\title{
Avaliação e Comparação da Hidrodinâmica e de Transferência de Oxigênio em Biorreator Convencional Agitado e Aerado e Biorreatores Pneumáticos.
}

\author{
R.G. de Oliveira ${ }^{2}$, M.O. Cerri ${ }^{1}$, \\ ${ }^{1}$ Universidade Estadual Paulista “Júlio de Mesquita Filho”, Faculdade de Ciências \\ Farmacêuticas, Departamento de Bioprocessos e Biotecnologia \\ ${ }^{2}$ Universidade Federal de São João Del Rei \\ E-mail para contato: marcel@fcfar.unesp.br
}

\begin{abstract}
RESUMO - Biorreatores são equipamentos utilizados na conversão de matériasprimas em algum produto de interesse. As reações que ocorrem dentro destes equipamentos são catalisadas por biocatalisadores, que podem ser enzimas ou células vivas. Um biorreator mantém o processo natural de crescimento dos microrganismos, oferendo as condições adequadas como temperatura, $\mathrm{pH}$, vitaminas, oxigênio e substrato. Os biorreatores mais utilizados no cultivo de microrganismos são os biorreatores convencionais tipo tanque agitado e aerado e os pneumáticos, tipo "coluna de bolhas", "Airlift" e "Split". Dessa forma, a transferência de oxigênio da fase gasosa para o liquido faz com que o oxigênio dissolvido chegue às células suspensas, penetrando-as e sendo consumido na reação. Contudo, alguns parâmetros devem ser analisados como critério de avaliação do desempenho de um biorreator, sendo o coeficiente volumétrico de transferência de oxigênio $\left(\mathrm{k}_{\mathrm{L}} \mathrm{a}\right)$ um importante parâmetro. $\mathrm{O}$ presente trabalho teve como objetivo mensurar os coeficientes volumétricos de transferência de oxigênio para os principais biorreatores (convencional e pneumático). Os resultados mostraram que os biorreator tipo "Airlift" e "coluna de bolhas" apresentaram coeficientes volumétricos de transferência de oxigênio próximos do biorreator convencional.
\end{abstract}

\section{INTRODUÇÃO}

Processos que ocorrem em condições de aerobiose se destacam dentre os processos biotecnológicos de interesse industrial, envolvendo o cultivo de células microbianas. A produção de enzimas, fermentos, antibióticos e vitaminas são exemplos. Segundo Schmidell et al. (2012) processos fermentativos envolvem o cultivo de células aeróbias facultativas ou aeróbias para a produção destes produtos citados ou mesmo para realizar tratamento biológico de águas residuais. Estes processos têm o comum aspecto de exigirem um dimensionamento adequado do sistema de transferência de oxigênio. 
Segundo Fazolo et al. (2001), o oxigênio é essencial no suprimento de energia para o metabolismo celular, em processos que envolvem o cultivo de células aeróbias ou aeróbias facultativas, portanto um dos parâmetros mais críticos na operação de um biorreator é, então, a transferência de oxigênio.

Segundo Cerri (2005) observa-se que um biorreator deve fornecer um ambiente adequado às células para que elas possam crescer e produzir os metabólitos de interesse. Embora existam diversos tipos de biorreatores, os convencionais, tipo tanque agitado e aerado e os biorreatores não convencionais, pneumáticos, tipo "coluna de bolhas" e tipo "Airlift" são os mais amplamente usado nos processos fermentativos. A utilização de um tipo de reator vai depender do tipo de experimento que se pretende realizar.

Para se realizar a comparação dos diferentes tipos de biorreatores e verificar o seu desempenho, alguns parâmetros devem ser analisados, dentre eles, o coeficiente volumétrico de transferência de oxigênio, a velocidade, tempo de circulação, retenção gasosa e a velocidade de cisalhamento.

O objetivo deste estudo, portanto, foi avaliar e comparar a transferência de oxigênio em quatro tipos de biorreatores: O Biorreator Convencional Agitado e Aerado e os Biorreatores Pneumáticos “Airlift”, "Coluna de Bolhas" e "Split".

\section{METODOLOGIA}

\subsection{Método Para Determinação Do $\mathbf{k}_{\mathrm{L}} \mathbf{a}$}

De acordo com Schmidell et al. (2012) o método dinâmico baseia-se em borbulhar nitrogênio no meio liquido a fim de que a concentração de oxigênio atinja o valor zero, quando isto ocorre, o fluxo de nitrogênio é então interrompido e inicia-se a aeração com uma vazão conhecida. A concentração de oxigênio aumenta com o tempo e uma curva de oxigênio em função do tempo é formada. Tratando-se essa curva e realizando o ajuste do modelo a dados experimentais é possível obter o valor do $\mathrm{k}_{\mathrm{L}} \mathrm{a}$. Há uma variação da concentração de oxigênio dissolvido (C) no tempo (t), a determinação do coeficiente volumétrico de transferência de oxigênio $\left(\mathrm{k}_{\mathrm{L}} \mathrm{a}\right)$ baseou-se no balanço de massa para o oxigênio na fase líquida, dada pela seguinte equação: 


$$
\frac{d C}{d t}=k_{L} a\left(C_{s}-C\right)
$$

Integrando-se a equação 1 e tomando-se como limite inferior de integração $\mathrm{C}=\mathrm{C}_{0}$ e $\mathrm{t}=\mathrm{t}_{0}$, obtém-se a equação 2 :

$$
C=C_{s}-\left(C_{s}-C_{0}\right) \cdot e^{-k L a \cdot(t-t 0)}
$$

Segundo Schmidell et al. (2012) para se corrigir esse atraso da sonda, foi proposto um modelo na qual o sinal da sonda $\left(\mathrm{C}_{\mathrm{e}}\right)$, varie no tempo proporcionalmente à diferença entre a concentração real de oxigênio $(\mathrm{C})$ e o sinal $\left(\mathrm{C}_{\mathrm{e}}\right)$, sendo $\mathrm{k}_{\mathrm{e}}$ a constante de atraso do eletrodo:

$$
\frac{\mathrm{dCe}}{\mathrm{dt}}=\mathrm{k}_{\mathrm{e}}\left(\mathrm{C}-\mathrm{C}_{\mathrm{e}}\right)
$$

Introduzindo na equação 3 o valor de $\mathrm{C}$ em função do tempo obtido na equação 2 , sendo $\mathrm{C}_{\mathrm{e}}=\mathrm{C}_{\mathrm{e} 0}$ e $\mathrm{t}=\mathrm{t}_{0}$, e integrando-se a equação resultante, obtém-se:

$$
C e=C_{e 0 .} e^{-k e(t-t 0)}+C e s .\left(1-e^{-k e(t-t 0)}\right)+\frac{k e .(\operatorname{Ces}-\operatorname{Co})}{K e-k_{L} a} \cdot\left(e^{-k e(t-t 0)}-e^{-k_{L} a(t-t 0)}\right)
$$

Ajustando-se a equação 4 aos dados experimentais da concentração de oxigênio dissolvido adquirido pelo eletrodo $\left(\mathrm{C}_{\mathrm{e}}\right)$ ao longo do tempo, e utilizando o algoritmo de Marquardt (com 95\% de confiança) foi possível obter o valor de $\mathrm{k}_{\mathrm{L}} \mathrm{a}$.

\subsection{Método Para Determinação da Retenção Gasosa}

Foram realizadas medidas visuais da altura do líquido não aerado e da altura da dispersão gás-líquido com o sistema aerado para a determinação da retenção gasosa. Foi utilizada a seguinte equação:

$$
\varepsilon_{G}=\frac{h_{D-h_{L}}}{h_{D}}
$$

Onde $h_{L}$ é altura do líquido não aerado e $h_{D}$ a altura da dispersão gás-líquido com o sistema aerado.

\subsection{Planejamento dos experimentos}

As medidas de $\mathrm{k}_{\mathrm{L}} \mathrm{a}$ e retenção gasosa foram realizadas em Biorreator Convencional da marca Marconi com quatro litros de volume de trabalho, diâmetro interno de 0,17 metros, quatro chicanas e equipado com um impelidor tipo turbina de Rushton. Medidas também foram realizadas no Biorreator Pneumático da marca Tecnal com cinco litros de volume de trabalho e diâmetro interno de 0,13 metros. Este segundo, operando como tipo "Airlift", tipo 
"Coluna de Bolhas" e "Split". Utilizando uma temperatura ambiente para o trabalho e variando a rotação no caso do convencional e a vazão de ar para todos os reatores foi possível realizar a comparação entre os biorreatores.

\section{RESULTADOS E DISCUSSÃO}

Os experimentos foram realizados em triplicatas com posterior tratamento estatístico. As tabelas 1, 2, 3, e 4 apresentam os valores de $\mathrm{k}_{\mathrm{L}} \mathrm{a}$ obtidos para os diferentes biorreatores estudados além de seus desvios padrões para um nível de confiança de 95\%. Observa-se por meio destas tabelas que dentre os biorreatores pneumáticos, o "Airlift" obteve maiores valores de $\mathrm{k}_{\mathrm{L}} \mathrm{a}$ em qualquer vazão aplicada e dentre o Convencional os maiores valores de $\mathrm{k}_{\mathrm{L}} \mathrm{a}$ foram obtidos com vazão de ar de 4 Litros/ min o que já era esperado. Comparado ao biorreator convencional, o biorreator "Airlift" apresentou valores de $\mathrm{k}_{\mathrm{L}} \mathrm{a}$ competitivos.

Pode-se observar pela tabela 4 que o biorreator convencional com uma vazão de 2 Litros/ min não obteve um maior valor de $\mathrm{k}_{\mathrm{L}} \mathrm{a}$ em uma rotação de aproximadamente $700 \mathrm{rpm}$. Isso pode ter ocorrido pelo fato do impelidor não ter transferido quantidade de movimento suficiente para o liquido, assim a transferência de oxigênio foi prejudicada para altos valores de vazão de ar. Este problema não ocorreu com o biorreator convencional com uma vazão de 4 Litros/min.

Tabela 1- Valores do $\mathrm{k}_{\mathrm{L}} \mathrm{a}$, altura de retenção, retenção gasosa, e o intervalo de confiança para as vazões específicas de ar para o biorreator "Airlift"

\begin{tabular}{|c|c|c|c|}
\hline $\begin{array}{c}\text { Vazão de } \\
\mathbf{a r} \\
\left(\mathbf{L . m i n}^{-\mathbf{1}}\right)\end{array}$ & $\begin{array}{c}\mathbf{k}_{\mathbf{L}} \mathbf{a} \\
\left(\mathbf{s}^{-\mathbf{1}}\right)\end{array}$ & $\begin{array}{c}\text { Retenção } \\
\text { Gasosa } \\
(\boldsymbol{\%})\end{array}$ & $\begin{array}{c}\text { Desvio- } \\
\text { Padrão } \\
\left(\mathbf{k}_{\mathbf{L}} \mathbf{a}\right)\end{array}$ \\
\hline 2,5 & 0,0069 & 1,32 & 0,0003 \\
\hline 5,0 & 0,0118 & 2,10 & 0,0008 \\
\hline 7,5 & 0,0146 & 3,24 & 0,0001 \\
\hline 10,0 & 0,0202 & 4,11 & 0,0001 \\
\hline 12,5 & 0,0223 & 5,33 & 0,0004 \\
\hline 15,0 & 0,0232 & 6,75 & 0,0009 \\
\hline 16,5 & 0,0238 & 7,67 & 0,0008 \\
\hline
\end{tabular}


Tabela 2 - Valores do $\mathrm{k}_{\mathrm{L}} \mathrm{a}$, altura de retenção, retenção gasosa, e o intervalo de confiança para as vazões específicas de ar para o biorreator "coluna de bolhas"

\begin{tabular}{|c|c|c|c|}
\hline $\begin{array}{c}\text { Vazão de ar } \\
\left(\mathbf{L}_{\mathbf{m i n}} \mathbf{- 1}^{\mathbf{1}}\right)\end{array}$ & $\begin{array}{c}\mathbf{k}_{\mathbf{L}} \mathbf{a} \\
\left(\mathbf{s}^{\mathbf{- 1}}\right)\end{array}$ & $\begin{array}{c}\text { Retenção } \\
\text { Gasosa } \\
(\boldsymbol{\%})\end{array}$ & $\begin{array}{c}\text { Desvio- } \\
\text { Padrão } \\
\left(\mathbf{k}_{\mathbf{L}} \mathbf{a}\right)\end{array}$ \\
\hline 2,5 & 0,0048 & 1,32 & 0,0001 \\
\hline 5,0 & 0,0095 & 2,36 & 0,0002 \\
\hline 7,5 & 0,0128 & 3,12 & 0,0004 \\
\hline 10,0 & 0,0160 & 4,11 & 0,0004 \\
\hline 12,5 & 0,0178 & 5,09 & 0,0005 \\
\hline 15,0 & 0,0209 & 6,05 & 0,0004 \\
\hline 16,5 & 0,0224 & 7,21 & 0,0007 \\
\hline
\end{tabular}

Tabela 3 - Valores do $\mathrm{k}_{\mathrm{L}} \mathrm{a}$, altura de retenção, retenção gasosa, e o intervalo de confiança para as vazões específicas de ar par ao biorreator "Split"

\begin{tabular}{|c|c|c|c|}
\hline $\begin{array}{c}\text { Vazão de } \\
\mathbf{a r} \\
\left(\mathbf{L} \cdot \mathbf{m i n}^{-\mathbf{1}}\right)\end{array}$ & $\begin{array}{c}\mathbf{k}_{\mathbf{L}} \mathbf{a} \\
\left(\mathbf{s}^{\mathbf{- 1}}\right)\end{array}$ & $\begin{array}{c}\text { Retenção } \\
\text { Gasosa } \\
(\boldsymbol{\%})\end{array}$ & $\begin{array}{c}\text { Desvio- } \\
\text { Padrão } \\
\left(\mathbf{k}_{\mathbf{L}} \mathbf{a}\right)\end{array}$ \\
\hline 2,5 & 0,0039 & 0,53 & 0,0001 \\
\hline 5,0 & 0,0072 & 1,32 & 0,0001 \\
\hline 7,5 & 0,0099 & 1,84 & 0,0001 \\
\hline 10,0 & 0,0126 & 2,61 & 0,0005 \\
\hline 12,5 & 0,0153 & 3,12 & 0,0006 \\
\hline 15,0 & 0,0177 & 4,11 & 0,0006 \\
\hline 16,5 & 0,0200 & 4,97 & 0,0002 \\
\hline
\end{tabular}


Tabela 4 - Valores de $\mathrm{k}_{\mathrm{L}} \mathrm{a}$, e o intervalo de confiança para biorreator convencional em diferentes condições de agitação e a aeração de 2 L.min ${ }^{-1}$ e de 4 L.min ${ }^{-1}$

\begin{tabular}{|c|c|c|c|c|c|}
\hline \multicolumn{3}{|c|}{ Vazão = 2 Litros/min } & \multicolumn{3}{c|}{ Vazão =4 Litros/min } \\
\hline $\begin{array}{c}\text { Rotação } \\
(\mathbf{r p m})\end{array}$ & $\begin{array}{c}\mathbf{k}_{\mathbf{L}} \mathbf{a} \\
\left(\mathbf{s}^{-\mathbf{1}}\right)\end{array}$ & Desvio-Padrão $\left(\mathbf{k}_{\mathbf{L}} \mathbf{a}\right)$ & $\begin{array}{c}\text { Rotação } \\
(\mathbf{r p m})\end{array}$ & $\begin{array}{c}\mathbf{k}_{\mathbf{L}} \mathbf{a} \\
\left(\mathbf{s}^{-1}\right)\end{array}$ & Desvio-Padrão $\left(\mathbf{k}_{\mathbf{L}} \mathbf{a}\right)$ \\
\hline 50 & 0,0023 & 0,0007 & 50 & 0,0024 & 0,0001 \\
\hline 100 & 0,0026 & 0,0003 & 100 & 0,0032 & 0,0002 \\
\hline 200 & 0,0070 & 0,0006 & 200 & 0,0077 & 0,0002 \\
\hline 300 & 0,0136 & 0,0001 & 300 & 0,0160 & 0,0001 \\
\hline 400 & 0,0158 & 0,003 & 400 & 0,0201 & 0,001 \\
\hline 500 & 0,0193 & 0,001 & 500 & 0,0216 & 0,002 \\
\hline 600 & 0,0196 & 0,002 & 600 & 0,0238 & 0,002 \\
\hline 700 & 0,0157 & 0,001 & 700 & 0,0264 & 0,003 \\
\hline
\end{tabular}

Com base nos valores de $\mathrm{k}_{\mathrm{L}} \mathrm{a}$ e das vazões obtidos na tabela acima, obtêm-se os gráficos mostrados nas figuras 1 e 2, onde é possível fazer uma comparação dos diferentes tipos de biorreatores estudados. Observa-se que o "Airlift" foi competitivo em relação à transferência de oxigênio.

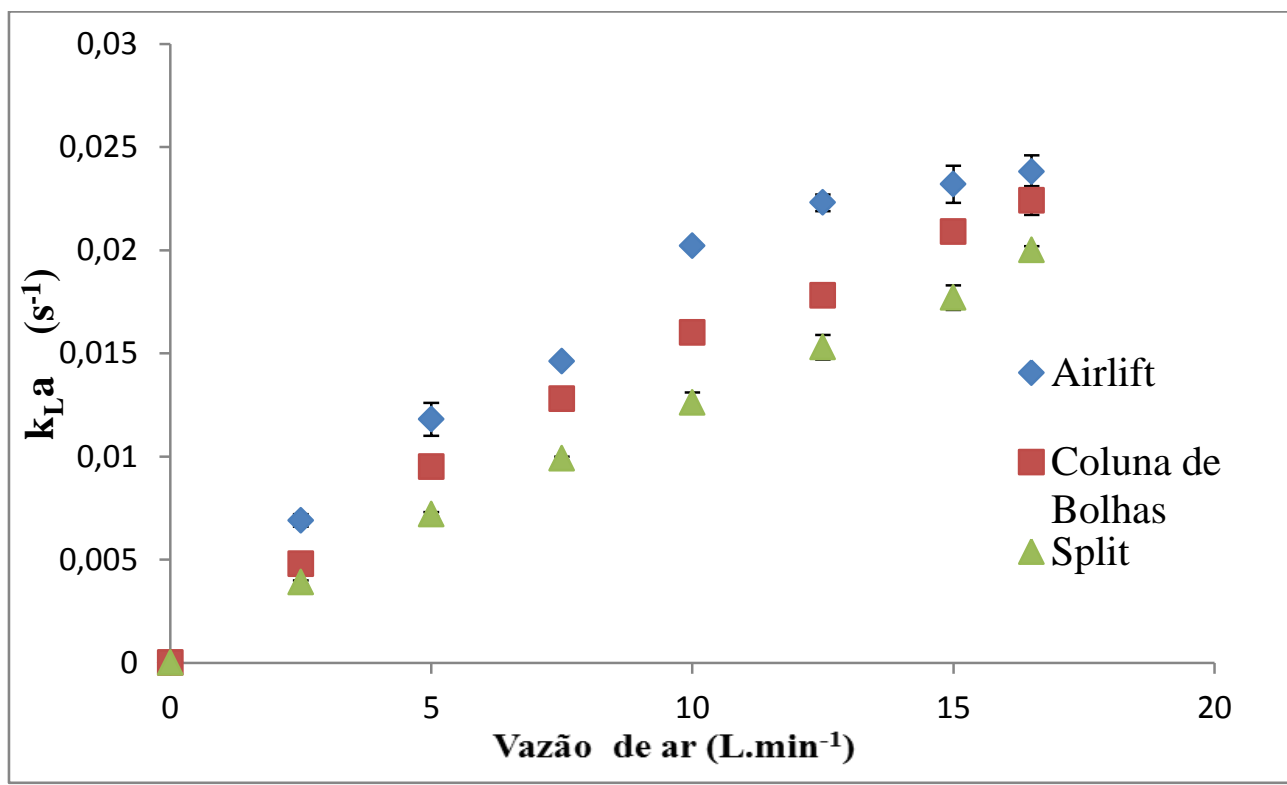

Figura 1 - Valores do $\mathrm{k}_{\mathrm{L}} \mathrm{a}$ em função das vazões de ar para os biorreatores pneumáticos "Ailift" Cilíndros Concêntricos, "Coluna de Bolhas" e "Split". 


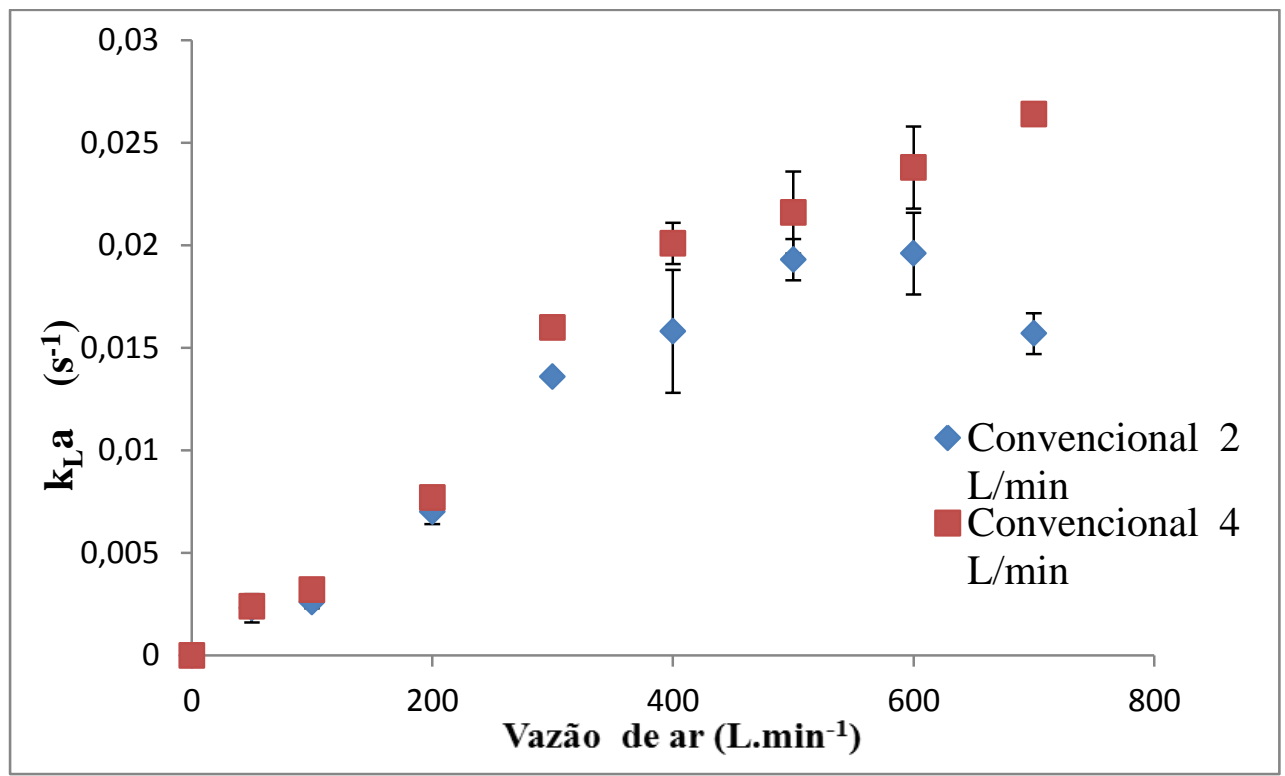

Figura 2 - Valores do $\mathrm{k}_{\mathrm{L}}$ a em função das rotações para os biorreatores convencionais nas vazões de 2 e 4 L.min ${ }^{-1}$.

Comparando com o trabalho de Thomasi (2010) a transferência de oxigênio dos biorreatores pneumáticos foram bastante próximas com o do presente trabalho. Para os biorreatores convencionais o trabalho de Campesi (2007) apresentou valores de $\mathrm{k}_{\mathrm{L}}$ a muito maiores do que o presente trabalho, isto pode ter ocorrido por causa da geometria do biorreator convencional Marconi, que não favoreceu a transferência de oxigênio da fase gasosa para a fase líquida.

\section{CONCLUSÕES}

Com base nos resultados obtidos, pode-se observar uma relação direta entre o coeficiente volumétrico de transferência de oxigênio e a vazão de ar, sendo que à medida que ar era injetado nos biorreatores a maior vazão, o valor do $\mathrm{k}_{\mathrm{L}}$ a aumentava.

Com relação aos biorreatores pneumáticos, o "Airlift" cilindros concêntricos obteve maiores valores de $\mathrm{k}_{\mathrm{L}} \mathrm{a}$ em qualquer vazão de ar comparado aos biorreatores "Split" e "Coluna de Bolhas”. Já com relação aos biorreatores convencionais, o analisado em uma maior vazão de $\operatorname{ar}\left(4\right.$ L. $\left.\min ^{-1}\right)$ obteve maiores valores de $\mathrm{k}_{\mathrm{L}} \mathrm{a}$ concluindo que a maior eficiência de transferência de oxigênio é influenciada pela maior vazão. Isso porque o impelidor com maior 
rotação se chocam com as bolhas fragmentando-as em tamanhos menores, aumentando assim, a transferência de oxigênio.

Comparando-se o "Airlift" aos biorreatores convencionais, percebe-se que estes apresentam valores competitivos, ou seja, o "Airlift" tem uma capacidade tão boa quanto o convencional de transferir oxigênio.

\section{REFERÊNCIAS}

SCHMIDELL, W; LIMA, U; AQUARONE, E; BORZANI, W. Biotecnologia industrial: Engenharia Bioquímica. São Paulo: E. Blücher, 2001. 2 v.

Cerri, M. O.; Avaliação de transferência de calor e massa de um biorreator airlift de circulação interna de bancada para a produção de ácido clavulânico, São Carlos - SP, Dissertação de mestrado, Universidade Federal de São Carlos, 2005.

Cerri, M. O.; Hidrodinâmica e transferência de oxigênio em três biorreatores airlift de circulação interna geometricamente semelhantes. Tese de Doutorado, Centro de Ciências e Tecnologia, UFSCar, São Carlos, SP. Brasil. 2009.

Fazolo, A.; Vieira, L. G. T.; Foresti, E.; Zaiat, M. Estimativa dos parâmetros cinéticos e de transferência de massa em um reator aeróbio de leito fixo utilizado no pós tratamento de efluente anaeróbio. In Pós-Tratamento de Efluentes de Reatores Anaeróbios- Coletânea de Trabalhos Técnicos, volume 2 (C A L Chernicharo, org.) pp. 203-218. Belo Horizonte: Segrac - PROSAB/FINEP, 2001

Thomasi S. S.; Hidrodinâmica e transferência de oxigênio em três biorreatores airlift de circulação interna geometricamente semelhantes. Dissertação de mestrado, Centro de Ciências e Tecnologia, UFSCar, São Carlos, SP. Brasil. 2010.

Campesi A.; Determinação da Velocidade de Cisalhamento Média em Biorreator Convencional Tipo Tanque Agitado e Aerado. Dissertação de mestrado, Centro de Ciências e Tecnologia, UFSCar, São Carlos, SP. Brasil. 2007. 